\title{
La Controvertida Participación del GLP-1 en los Mecanismos Fisiológicos Desencadenados tras Cirugía Bariátrica
}

\author{
The Controversial Participation of GLP-1 in the Physiological \\ Mechanisms Unchained After Bariatric Surgery
}

\author{
Joshua Falckenheiner-Soria'; José Bancalero-De Los Reyes ${ }^{3}$; Alejandra Moreno-Arciniegas ${ }^{4}$; \\ Alonso Camacho-Ramírez ${ }^{4}$ J. Arturo Prada-Oliveira² \& Gonzalo M. Pérez-Arana²
}

\begin{abstract}
FALCKENHEINER-SORIA, J.; BANCALERO-DE LOS REYES, J.; MORENO-ARCINIEGAS, A.; CAMACHO-RAMÍREZ, A.; PRADA-OLIVEIRA, J. A. \& PÉREZ-ARANA, G. M. La controvertida participación del GLP-1 en los mecanismos fisiológicos desencadenados tras cirugía bariátrica. Int. J. Morphol., 37(1):76-81, 2019.
\end{abstract}

RESUMEN: Numerosas hipótesis se invocan para explicar el efecto beneficioso sobre el metabolismo glucídico tras la cirugía bariátrica. Algunos autores abogan por la secreción y liberación de distintas sustancias con funciones endocrinas (enterohormonas). Una de las sustancias más señaladas como efector, con efectos contrastados pero datos controvertidos, es el GLP-1. Nuestro estudio se realizó en ratas Wistar macho sanas, para evitar la ausencia de factores de confusión como son la DMT2 y la obesidad. Para conocer el mapa de adaptación a la secreción de GLP-1 tras la cirugía, se designaron 5 grupos: dos grupos control (de ayuno y de estrés quirúrgico); y tres grupos quirúrgicos (gastrectomía vertical, resección del $50 \%$ del intestino medio y el Bypass gástrico con montaje en Y de Roux). Después de tres meses se estudiaron mediante técnicas inmunohistoquímicas el patrón de síntesis de GLP-1 en las distintas porciones del intestino delgado. También se estudió la expresión de los receptores de membrana en las células de los islotes pancreáticos. Se observó la existencia de un significativo aumento del número de células secretoras en íleon, duodeno y yeyuno en los grupos quirúrgicos de técnicas mixtas (RYGB) y malabsortivas (RI50). Igualmente se observó una elevación de los receptores pancreáticos en las mismas técnicas frente a los controles. Nuestros datos indican que la secreción intestinal de GLP-1 y su sensibilidad a nivel pancreáticas están aumentada, como efecto adaptativo a la agresión mecánica del tubo y a la alteración del flujo de nutrientes tras la cirugía.

PALABRAS CLAVE: Páncreas; Diabetes; Bariatric-surgery; Insulin-Secreting-Cells; Enterohormonas.

\section{INTRODUCTION}

La cirugía bariátrica (CB) ha abierto un nuevo campo en el tratamiento de la diabetes mellitus tipo 2 (DMT2), gracias a la remisión de un porcentaje importante de pacientes intervenidos por obesidad (Pories et al., 1995; Buchwald et al., 2009). Bajo el nombre de CB se agrupan distintas entidades con distintas afectaciones anatómico-funcionales y distintos resultados en la resolución de la DMT2 (de Gordejuela et al., 2011; Mingrone et al., 2012). Dentro de estas cabe destacar la gastrectomía vertical (GV) como técnica restrictiva y el Bypass gástrico en Y de Roux (RYBG) como técnica mixta (con componente restrictivo y malabsortivo).

Se han publicado numerosos estudios relativos, tanto en humanos como en animales de experimentación, que intentan dilucidar los mecanismos íntimos que llevan a esta resolución de la DMT2 tras CB. Si bien parece claro que el eje central gira en torno al triángulo conformado por el tubo digestivo, el páncreas y los tejidos periféricos que gestionan la resistencia periférica a la insulina (hígado, grasa visceral y músculo), no está en absoluto claro cuáles son los agentes implicados en la regulación de las interacciones entre estos tres vértices (Yousseif et al., 2014; Penney et al., 2015). Numerosos estudios apuntan a determinadas enterohormonas, que secretadas por distintas porciones del tubo digestivo, poseen implicaciones metabólicas a todos los niveles orgánicos. Uno de los efectores más invocado es el caso del Glucagon-like peptide-1 (GLP-1), secretado por las células L del íleon. Se le atribuye la capacidad de estimular la proliferación de las células b en el páncreas y au-

\footnotetext{
${ }^{1}$ Virgen de las Montañas Hospital, Hospitales JM Pascual y Pascual, Cádiz, Spain.

${ }^{2}$ Department of Human Anatomy and Embryology, Faculty of Medicine, Universitary of Cádiz, Spain.

${ }^{3}$ Complejo Hospitalario de Badajoz, Servicio Extremeño de Salud, Badajoz, Spain.

${ }^{4}$ Department of Surgery, Puerto Real Universitary Hospital, University of Cádiz, Cádiz, Spain.
} 
FALCKENHEINER-SORIA, J.; BANCALERO-DE LOS REYES, J.; MORENO-ARCINIEGAS, A.; CAMACHO-RAMÍREZ, A.; PRADA-OLIVEIRA, J. A. \& PÉREZ-ARANA, G. M. La controvertida participación del GLP-1 en los mecanismos fisiológicos desencadenados tras cirugía bariátrica. Int. J. Morphol., 37(1):76-81, 2019.

mentar la secreción de insulina por parte de estas (Liu et al., 2011; Yabe \& Seino, 2011). Así como de reducir la sensibilidad a la misma en los tejidos periféricos e hígado (Tian \& Jin, 2016). Por esto se le ha considerado un elemento clave en la resolución de la DMT2, dado que actúa sobre los dos elementos patogénicos de la misma: la resistencia periférica y el daño sobre la célula $b$.

Sin embargo, este papel del GLP-1 no está completamente aceptado. Otros autores han mostrado en modelos Knockout para GLP-1, la resolución de DMT2 tras la realización de RYGB (Mokadem et al., 2013). Así mismo, estudios realizados en ratones Knockout para el gen del receptor pancreático del GLP-1 (GLP-1r), mostraron ausencia de modificaciones en relación a la cirugía de GV (Wilson-Pérez et al., 2013).

Nuestro estudio pretende ahondar en el conocimiento sobre el GLP-1. Utilizando un modelo de roedor sano, nos centramos en la determinación de las zonas de secreción de GLP-1 en el intestino, tras la realización de técnicas de CB. Por otro lado, determinamos la sensibilidad a esta enterohormona en la población celular b pancreática, a través de la expresión de su receptor (GLP-1r). El propósito es comprobar los cambios que se producen en este eje enteropancreático tras dos variantes de CB (la GV y el RYBG) y una tercera técnica experimental malabsortiva que implica la resección del $50 \%$ del intestino delgado (RI50).

\section{MATERIAL Y MÉTODO}

Animales. El mantenimiento, estabulación, los procedimientos quirúrgicos y sacrificio de los animales se realizaron con la aprobación del comité de ética para animales de experimentación de la Universidad de Cádiz, España. Los animales se mantuvieron bajo condiciones normalizadas, en un ambiente controlado $\left(21^{\circ} \mathrm{C}\right.$, con ciclos de luz, disposición de agua y dieta estándar ad libitum). Para el estudio se utilizaron ratas Wistar macho de $250 \mathrm{~g}$ divididas aleatoriamente en cinco grupos $(n=6)$ : un control de ayuno (CA); un control quirúrgico (SHAM); la resección intestinal del $50 \%$ (RI50); Bypass Gástrico en Y de Roux (RYBG) y gastrectomía vertical (GV).

\section{Procedimientos quirúrgicos.}

Grupos controles. En el grupo (CA) los animales se sometieron a un periodo de ayuno de $24 \mathrm{~h}$ pre- y post-quirúrgico, igual al que se sometieron a los demás grupos. En el caso de los animales del grupo (SHAM), tras la inducción anestésica con Isofluorano (Isoflo, Abbott 571329.8) se llevó a cabo una pequeña laparotomía media de unos $2-3 \mathrm{~cm}$ en el tercio medio de la línea media abdominal, la evisceración de todas las asas de intestino delgado y sección transversal completa a nivel de intestino medio sin resección del mismo. La anastomosis de las asas se hizo termino-terminal, con puntos sueltos (Vicryl $\left.{ }^{\circledR} 4 / 0\right)$. Las asas de intestino se reintrodujeron y se cerró la pared abdominal en un solo plano (Vicryl ${ }^{\circledR} 3 / 0$ ) (Pérez-Arana et al., 2015; Camacho-Ramírez et al., 2017).

Grupos experimentales. En el grupo (RI50) se realizaron los pasos descritos para el grupo (SHAM) y una vez expuestas las asas del intestino delgado se midió la distancia entre la flexura duodenoyeyunal (ángulo de Treitz) y la papila ileal (válvula ileocecal). Se resecó $50 \%$ de intestino delgado, preservando el $25 \%$ del intestino delgado distal a la flexura duodenoyeyunal y el $25 \%$ proximal a la papila ileal. El resto del procedimiento se realizó de manera idéntica.

En el grupo RYBG tras laparotomía media, se realizó una evisceración y una sección transversa del estómago con tijeras, previa aplicación de clamps atraumáticos entre las curvaturas mayor a menor. Se cerró mediante sutura continua (Prolene $\AA 4 / 0$ ), menos $0.5 \mathrm{~cm}$ para restablecer la continuidad al asa alimentaria. La sección transversa del yeyuno, a $10 \mathrm{~cm}$ distal a la flexura duodenoyeyunal, se anastomosó al remanente gástrico superior. La continuidad del tubo digestivo se configuró con la anastomosis entre el asa alimentaria ( $10 \mathrm{~cm}$ de longitud desde la anastomosis gastroyeyunal) con el asa biliar, mediante anastomosis terminolateral (Prolene ${ }^{\circledR 4 / 0)}$. El resto del procedimiento se realizó de manera idéntica que en el grupo anterior.

El grupo GV se realizó una laparotomía media, la gastrolisis del omento menor y la sección entre ligaduras del ligamento gastro-esplénico. Para la resección de la curvatura mayor se aplicó una pinza curva atraumática englobando todo el estómago. Se preservó tanto el píloro como el cardias (unión esófago-gástrica), resecándose la mayor parte del fundus gástrico, cuerpo gástrico, curvatura mayor y antro pilórico. Se realizó una gastrorrafia para la estanqueidad completa del estómago. Los últimos pasos de la intervención fueron comunes a los otros grupos (PérezArana et al., 2015; Camacho-Ramírez et al.).

Sacrificio, extracción y procesamiento de muestras. Transcurridas 12 semanas desde la intervención se procedió a realizar el sacrificio de los animales. Se llevó a cabo con hidrato de cloral intraperitoneal. Tras prefijación, se procedió a la extracción del páncreas y porciones de tubo digestivo (antro pilórico, duodeno, yeyuno e íleon). La postfijación se hizo por inmersión en solución de Bouin a $4{ }^{\circ} \mathrm{C}$ durante $24 \mathrm{~h}$. Las piezas obtenidas fueron deshidratadas e incluidas en parafina, para ser después cortadas en un micrótomo en secciones de 8 a $10 \mu \mathrm{m}$. 
Estudio mediante inmunofluorescencia de la expresión de GLP-1 en tubo digestivo. Cortes de tejido intestinal procedente de los tres segmentos de intestino delgado, de todos los grupos estudiados, fueron rehidratados, permeabilizados e incubados con un anticuerpo primario anti-GLP-1 de ratón, (Abcam, Cambridge, CB4 UK) 12 h a $4{ }^{\circ} \mathrm{C}$. Posteriormente los cortes fueron revelados con un anticuerpo secundario anti $\mathrm{IgG}$ de ratón conjugado con un fluoróforo Alexa, 488 (Molecular Probes Inc Eugene, USA). Los núcleos celulares fueron teñidos con 4'-6’Diamino fenil indol (DAPI). Las muestras obtenidas fueron valoradas por un solo observador, con un microscopio de fluorescencia equipado con una cámara digital (Olympus DP71), asociada con el software Cell-D ${ }^{\circledR}$. Se analizaron 8 cuadrantes en cada punto a estudio, expresándose el resultado como número de células GLP-1 positivas $/ \mathrm{mm}^{2}$ de superficie de tubo digestivo.

Estudio de expresión de GLP-1r en páncreas. Los cortes de páncreas de cada uno de los grupos fueron rehidratados, permeabilizados e incubados con un anticuerpo $\mathrm{IgG}$ frente GLP-1r de conejo (Abcam, Cambridge, CB4 OFL UK) y teñidos con un anticuerpo de ratón IgG anti-insulina (SigmaAldrich, I-2018 USA), 12 h a $4{ }^{\circ} \mathrm{C}$. Posteriormente contra los anticuerpos primaros, se usaron anticuerpos anti IgG de conejo y anti IgG de ratón conjugados con fluoróforos Alexa, 546 y Alexa 488 (Molecular Probes Inc Eugene, USA). Los núcleos celulares fueron teñidos con 4'-6’Diamino-fenilindol (DAPI). Las muestras obtenidas fueron valoradas por un solo observador con un microscopio de fluorescencia y cámara digital (Olympus DP71), asociada con el software Cell-D®. Se analizaron 15 islotes pancreáticos al azar en cada punto a estudio, expresándose el resultado como número de células $\mathrm{b}$ positivas/GLP-1r positivas $/ \mathrm{mm}^{2}$ islote pancreático.

Estudio estadístico. Los datos obtenidos tras el análisis estadístico de los resultados se expresan como valores medios +eem de las mediciones realizadas en $n=6$ animales por grupo. Las comparaciones entre los distintos grupos fueron realizadas mediante el test ANOVA, considerándose diferencias significativas aquellas con $\mathrm{P}<0,05$. Para el estudio se utilizó el paquete informático SPSS versión 24.0.

\section{RESULTADOS}

Expresión de GLP-1 en el tubo digestivo. Los datos obtenidos del estudio de la expresión de GLP-1 en la mucosa del intestino delgado se encuentran representados para los tres segmentos intestinales estudiados duodeno, yeyuno e íleon (Figs. 1, 2 y 3). En ninguno de los casos aparecieron dife-

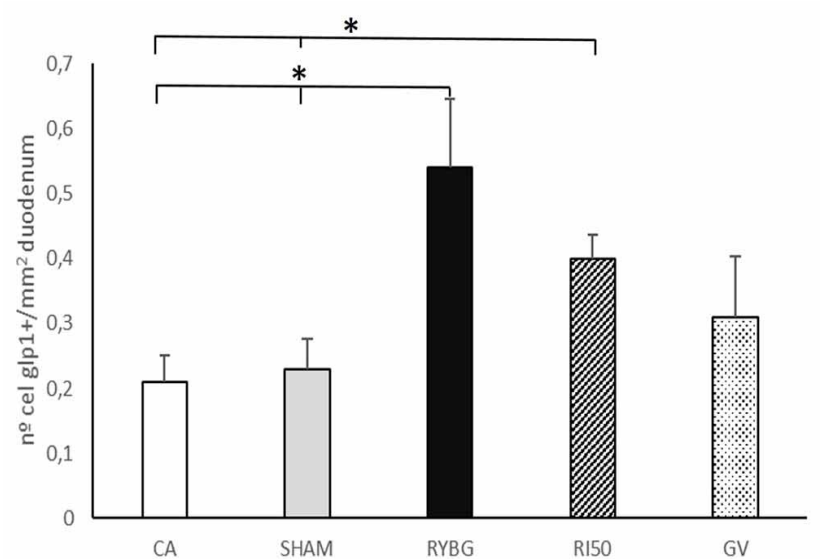

Fig. 1. Representamos en el eje de ordenadas el número de células en el duodeno GLP-1 positivas $/ \mathrm{mm}^{2}$ expresadas como valores medios + e.e.m, para cada uno de los cinco grupos a estudio, representados en el eje de abscisas. Se aprecian diferencias significativas entre ambos grupos control CA y Sham y los grupos RYGB y RI50. $(0,21+0,04 ; 0,23+0,04$ Vs $0,54+0,10 ; 0,40+0,03)$ para $\mathrm{P}<0,05$.

rencias significativas entre ambos grupos control CA y Sham. En el caso del duodeno podemos observar incrementos significativos en RI50 y RYGB en el número de células GLP1 positivas $/ \mathrm{mm}^{2}$ respecto a ambos grupos control CA y Sham (Fig. 1). En la expresión de células GLP-1 en el yeyuno el incremento significativo respecto a ambos grupos control CA y Sham solo aparece en el grupo RYGB. Los valores representados para el grupo RI50 son referidos al estudio de la porción proximal remanente tras la resección (Fig. 2).

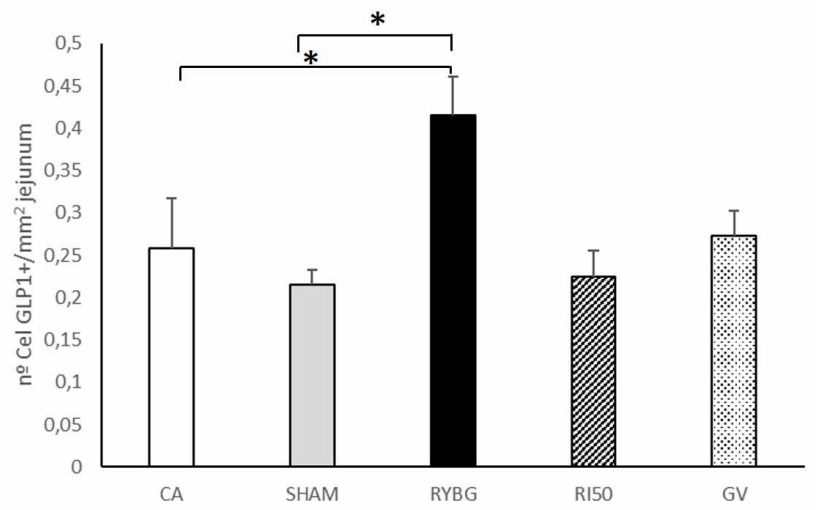

Fig 2. Representamos en el eje de ordenadas el número de células en el yeyuno GLP-1 positivas/mm² expresadas como valores medios + e.e.m para cada uno de los 5 grupos a estudio representados en el eje de abscisas. Se aprecian diferencias significativas entre ambos grupos control CA y Sham y el grupo RYGB. $(0,25+0,12$; $0,21+0,03$, Vs $0,41+0,09)$ para $\mathrm{P}<0,05$.

En el íleon se registran incrementos significativos del número de células GLP-1 positivas $/ \mathrm{mm}^{2}$ respecto a ambos grupos control CA y Sham tanto en el grupo RI50 como en el RYGB (Fig. 3). 


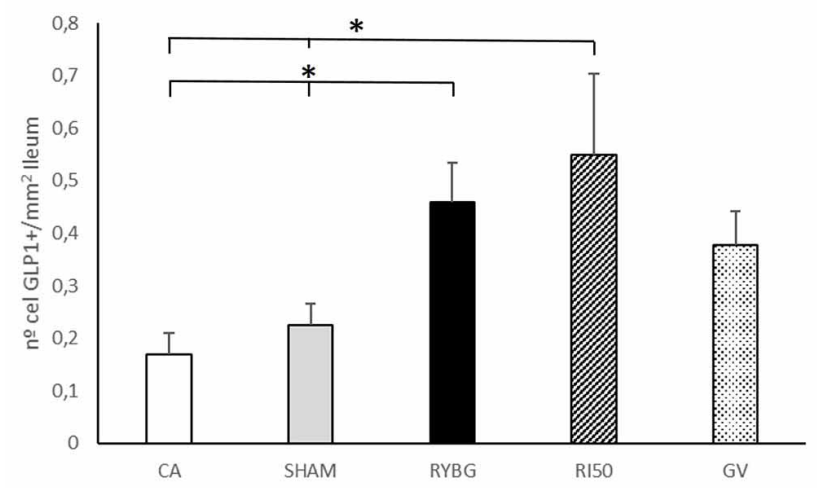

Fig 3. Representamos en el eje de ordenadas el número de células en el íleon GLP-1 positivas $/ \mathrm{mm}^{2}$ expresadas como valores medios + e.e.m para cada uno de los 5 grupos a estudio representados en el eje de abscisas. Se aprecian diferencias significativas entre ambos grupos control CA y Sham y los grupos RYGB y RI50. $(0,17+0,04$; $0,22+0,04$ Vs $0,46+0,07 ; 0,55+0,15)$ para $\mathrm{P}<0,05$.

Expresión del GLP-1r en la población celular pancreática. Los datos obtenidos del estudio de la expresión de receptores para el GLP-1 en las células b de los islotes pancreáticos muestran un incremento significativo en el número de células b GLP-1r positivas $/ \mathrm{mm}^{2}$ de islote pancreático en el grupo RYGB respecto a ambos grupos control (CA y Sham). Este incremento respecto a los controles se acentúa más si cabe en el grupo RI50 (Fig. 4).

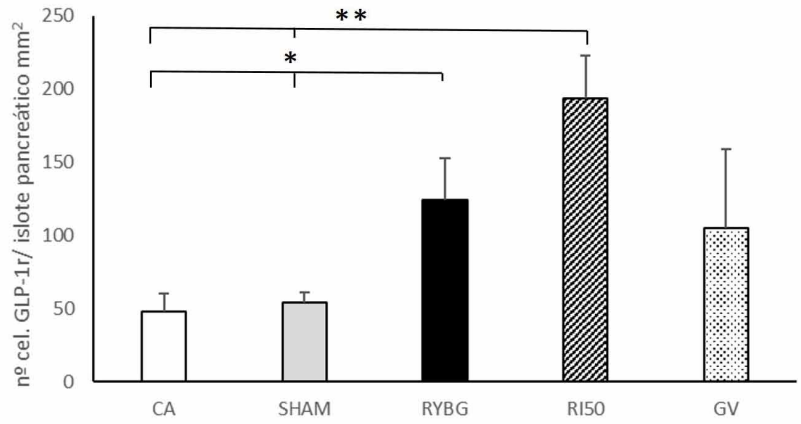

Fig 4. En esta grafica podemos ver representado en el eje de ordenadas el número de células b GLP-1r positivas $/ \mathrm{mm}^{2}$ de islote pancreático expresadas como valores medios + e.e.m, para cada uno de los 5 grupos a estudio representados en el eje de abscisas. Se aprecian diferencias significativas entre ambos grupos control CA y Sham y los grupos RYGB y RI50. $(48,01+12,65 ; 54,06+7,21$ Vs $124,45+28,55$; $193,73+29,04)$ para $\mathrm{P}<0,05$ y $\mathrm{P}<0,01$ respectivamente.

\section{DISCUSIÓN}

Tanto el GLP-1 como el PYY -ambos secretados en las células L del íleon- o la Grelina - en el antro- han sido involucrados tanto en mecanismos fisiológicos como fisiopatológicos, en el llamado eje entero-pancreático que llevan a la resolución de la DMT2 (Thaler \& Cummings, 2009; Batterham \& Cummings, 2016).

En este sentido, numerosos trabajos han realizado determinaciones de los niveles plasmáticos de GLP-1. Observando la mayoría elevaciones de estos tras la realización de RYGB o GV, coincidentes con mejoras en la tolerancia a la glucosa, tanto en humanos como en animales (Chambers $e t$ al., 2014; Yousseif et al.). Pero son escasos los estudios que de manera directa analizan la variación de la secreción de esta hormona en el mismo tejido secretor tras la realización de este tipo de intervenciones, y ninguno que de forma exhaustiva analice otras zonas del tubo digestivo distintas a su lugar de síntesis establecido (íleon).

Aun así, han sido de gran aceptación algunas teorías que proponían como principal motivo para la resolución de la DMT2 el incremento de liberación de GLP-1 exclusivamente en el íleon, tras la llegada temprana de los nutrientes tras estas cirugías (Hindgut Hypothesis) (Nausheen et al., 2013).

Los hallazgos obtenidos en el presente estudio nos hacen pensar que estas teorías pueden ser acertadas solo en parte. En nuestro estudio observamos que tras la realización de RYGB, no solo se incrementa la expresión de GLP-1 en el íleon (Fig. 3), sino que también aparecen incrementos en su expresión en el yeyuno y en duodeno (Figs. 1 y 2). Estos resultados corroborarían el importante papel de GLP-1, activándose puntos de secreción alternativos en el tubo digestivo tras la agresión mecánica de la cirugía y, probablemente, tras las adaptaciones metabólicas subsiguientes a la misma.

Pero de otro lado cuestionan la llegada temprana de productos de la digestión o la presencia de elementos sin digerir en el íleon como factor desencadenante del incremento de la expresión de GLP-1. Más aún si pensamos en el incremento de expresión de GLP-1 en el duodeno de nuestro modelo tras RYGB, que permanece excluido de dicho tránsito, por tanto no podrían ser los nutrientes los efectores de ese estímulo secretor. También aparece elevado en el duodeno, en los animales sometidos a RI50, en los cuales el transito duodenal es normal (Fig. 1).

Ante esto cabe pensar en la posibilidad de que GLP1 no sea el primer eslabón en una cadena de incretinas que regulan el eje entero-pancreático. Es posible que la expresión de GLP-1 esté mediada por otras hormonas; y que esta estuviera estimulada por la llegada temprana de productos de la digestión o la presencia de elementos sin digerir en el íleon. En este sentido existen trabajos que denotan un aumento en los niveles postprandiales de PYY, secretado por las células L del íleon en pacientes intervenidos de RYGB 
FALCKENHEINER-SORIA, J.; BANCALERO-DE LOS REYES, J.; MORENO-ARCINIEGAS, A.; CAMACHO-RAMÍREZ, A.; PRADA-OLIVEIRA, J. A. \& PÉREZ-ARANA, G. M. La controvertida participación del GLP-1 en los mecanismos fisiológicos desencadenados tras cirugía bariátrica. Int. J. Morphol., 37(1):76-81, 2019.

(Chronaiou et al., 2012; Yousseif et al.). Este dato podría señalarlo como un posible candidato a ser la incretina que inicie este mecanismo.

En el otro extremo del eje entero-pancreático, nos encontramos también con modificaciones en la sensibilidad a GLP-1 de la población celular b evidenciamos un incremento significativo de la expresión del receptor para el GLP1 en los páncreas de los animales sometidos a RYGB y RI50, respecto a sus controles (Fig. 4). Estos datos estarían en consonancia con estudios que señalan incrementos en la población de células b tras esta cirugía; así como la capacidad de GLP-1 para modular las funciones de la masa b pancreática (Kubo et al., 2016; Camacho-Ramírez et al.).

Analizando este resultado teniendo en cuenta las técnicas quirúrgicas, si nos planteamos que relación se establece con la porción de tubo afectado, observamos que este incremento del GLP-1r se da en los animales que han sufrido un cambio anatomofuncional que conlleva la llegada temprana de productos de la digestión al íleon (RYGB y RI50); excluyéndose el grupo sometido a GV. Esto nos lleva a pensar nuevamente en el PYY, sobre todo a raíz de estudios recientes que ponen de manifiesto su capacidad regulatoria de las funciones de las células b humanas o de islotes murinos en cultivo (Khan et al., 2016).

Otra posible explicación a ser el factor desencadenante tanto del incremento de la expresión de GLP1 en el tubo digestivo como del aumento de la sensibilidad a este en los islotes pancreáticos podría ser el denominado factor anti-incretina (Rubino \& Marescaux, 2004). Este factor fue propuesto por algunos trabajos como responsable de la mejora del metabolismo de la glucosa tras la realización de RYGB en ratas y humanos, debido a la exclusión del duodeno y yeyuno proximal al paso de los nutrientes (Rubino et al., 2006; Pacheco et al., 2007).

No obstante, si atendemos a los resultados mostrados, el incremento de síntesis de GLP-1 y de GLP-1r en el páncreas que aparece en el grupo RI50, que no presenta dicha exclusión a dichos nutrientes (Figs. 1 y 4). Lo que nos hace dudar acerca de este "factor anti-incretina".

Proponemos un acercamiento a los mecanismos fisiopatológicos que gobiernan el eje entero-pancreático tras cada uno de los tipos de CB. Concluimos el importante papel de la incretina GLP-1, cuya expresión no se limita al íleon sino que aparece incrementada en duodeno y yeyuno tras algunos tipo de intervenciones. Así mismo el GLP-1 no parece tener un papel inicial en los cambios en el eje enteropancreático, sino que podría situarse en un escalón intermedio. Probablemente determinado por otras incretinas, tal vez el PYY a tenor de los indicios publicados. En cualquier caso, nuestro trabajo muestra que el GLP-1 ha de jugar un papel fundamental en los cambios fisiológicos en el eje enteropancreático tras la $\mathrm{CB}$.

FALCKENHEINER-SORIA, J.; BANCALERO-DE LOS REYES, J.; MORENO-ARCINIEGAS, A.; CAMACHORAMÍREZ, A.; PRADA-OLIVEIRA, J. A. \& PÉREZ-ARANA, G. M. The controversial participation of GLP-1 in the physiological mechanisms unchained after bariatric surgery. Int J. Morphol., 37(1):76-81, 2019.

SUMMARY: Numerous hypotheses are invoked to explain the beneficial effect on glucose metabolism after bariatric surgery. Some authors advocate for the secretion and release of various substances with endocrine functions (enterohormones). One of the substances most marked as effector, with contrasting effects but controversial data, is Glucagon-like peptide-1 GLP-1. Our study was performed in healthy male Wistar rats, to avoid the absence of confounding factors such as DMT2 and obesity. In order to know the map of adaptation to GLP-1 secretion after surgery, five groups were designated: Two control groups (fasting and surgical stress); and three surgical groups (vertical sleeve gastrectomy, $50 \%$ midgut resection and Roux-en-Y gastric bypass). After three months, the GLP-1 synthesis pattern was studied by immunohistochemical techniques in the different portions of the small digestive tract. The expression of membrane receptors in pancreatic islet cells was also studied. There was a significant increase in the number of secretory cells in ileum, duodenum and jejunum in mixed surgical (RYGB) and malabsorptive (RI50) groups. An elevation of pancreatic receptors was also observed in the same techniques against controls. Our data indicated that intestinal secretion of GLP1 and its sensitivity to the pancreatic level were increased, both to an adaptive effect to the mechanical aggression of the digestive tube and to the alteration of nutrient flow after surgery.

KEY WORDS: Pancreas: Diabetes; Bariatric-surgery; Insulin-Secreting Cells; Enterohormones.

\section{REFERENCIAS BIBLIOGRÁFICAS}

Batterham, R. L. \& Cummings, D. E. Mechanisms of diabetes improvement following bariatric/metabolic surgery. Diabetes Care, 39(6):893-901, 2016.

Buchwald, H.; Estok, R.; Fahrbach, K.; Banel, D.; Jensen, M. D.; Pories, W. J.; Bantle, J. P. \& Sledge, I. Weight and type 2 diabetes after bariatric surgery: systematic review and meta-analysis. Am. J. Med., 122(3):24856, 2009.

Camacho-Ramírez, A.; Blandino-Rosano, M.; Segundo-Iglesias, M. C.; Lechuga-Sancho, A. M.; Aguilar-Diosdado, M.; Pérez-Arana, G. M. \& Prada-Oliveira, J. A. Bariatric surgery influences b-cell turnover in non obese rats. Histol. Histopathol., 32(12):1341-50, 2017.

Chambers, A. P.; Smith, E. P.; Begg, D. P.; Grayson, B. E.; Sisley, S.; Greer, T.; Sorrell, J.; Lemmen, L.; LaSance, K.; Woods, S. C.; Seeley, R. J.; D'Alessio, D. A. \& Sandoval, D. A. Regulation of gastric emptying 
rate and its role in nutrient-induced GLP-1 secretion in rats after vertical sleeve gastrectomy. Am. J. Physiol. Endocrinol. Metab., 306(4):E424-32, 2014

Chronaiou, A.; Tsoli, M.; Kehagias, I.; Leotsinidis, M.; Kalfarentzos, F. \& Alexandrides, T. K. Lower ghrelin levels and exaggerated postprandial peptide-YY, glucagon-like peptide-1, and insulin responses, after gastric fundus resection, in patients undergoing Rouxen-Y gastric bypass: a randomized clinical trial. Obes. Surg., 22(11):1761-70, 2012.

de Gordejuela, A. G.; Pujol Gebelli, J.; García, N. V.; Alsina, E. F.; Medayo, L. S. \& Masdevall Noguera, C. Is sleeve gastrectomy as effective as gastric bypass for remission of type 2 diabetes in morbidly obese patients? Surg. Obes. Relat. Dis., 7(4):506-9, 2011.

Khan, D.; Vasu, S.; Moffett, R. C.; Irwin, N. \& Flatt, P. R. Islet distribution of Peptide YY and its regulatory role in primary mouse islets and immortalised rodent and human beta-cell function and survival. Mol. Cell. Endocrinol., 436:102-13, 2016.

Kubo, F.; Miyatsuka, T.; Sasaki, S.; Takahara, M.; Yamamoto, Y.; Shimo, N.; Watada, H.; Kaneto, H.; Gannon, M.; Matsuoka, T. A. \& Shimomura, I. Sustained expression of GLP-1 receptor differentially modulates b-cell functions in diabetic and nondiabetic mice. Biochem. Biophys. Res. Commun., 471(1):68-74, 2016.

Liu, Y.; Zhou, Y.; Wang, Y.; Geng, D. \& Liu, J. Roux-en-Y gastric bypassinduced improvement of glucose tolerance and insulin resistance in type 2 diabetic rats are mediated by glucagon-like peptide-1. Obes. Surg., 21(9):1424-31, 2011.

Mingrone, G.; Panunzi, S.; De Gaetano, A.; Guidone, C.; Iaconelli, A.; Leccesi, L.; Nanni, G.; Pomp, A.; Castagneto, M.; Ghirlanda, G. \& Rubino, F. Bariatric surgery versus conventional medical therapy for type 2 diabetes. N. Engl. J. Med., 366(17):1577-85, 2012.

Mokadem, M.; Zechner, J. F.; Margolskee, R. F.; Drucker, D. J. \& Aguirre, V. Effects of Roux-en-Y gastric bypass on energy and glucose homeostasis are preserved in two mouse models of functional glucagon-like peptide-1 deficiency. Mol. Metab., 3(2):191-201, 2013.

Nausheen, S.; Shah, I. H.; Pezeshki, A.; Sigalet, D. L. \& Chelikani, P. K. Effects of sleeve gastrectomy and ileal transposition, alone and in combination, on food intake, body weight, gut hormones, and glucose metabolism in rats. Am. J. Physiol. Endocrinol. Metab., 305(4):E50718, 2013.

Pacheco, D.; de Luis, D. A.; Romero, A.; González Sagrado, M.; Conde, R.; Izaola, O.; Aller, R. \& Delgado, A. The effects of duodenal-jejunal exclusion on hormonal regulation of glucose metabolism in GotoKakizaki rats. Am. J. Surg., 194(2):221-4, 2007.

Penney, N. C.; Kinross, J.; Newton, R. C. \& Purkayastha, S. The role of bile acids in reducing the metabolic complications of obesity after bariatric surgery: a systematic review. Int. J. Obes. (Lond.), 39(11):1565-74, 2015.

Pérez-Arana, G.; Camacho-Ramírez, A.; Segundo-Iglesias, M. C.; Lechuga-Sancho, A. M.; Sancho-Maraver, E.; Aguilar-Diosdado, M. \& Prada-Oliveira, J. A. A surgical model of short bowel syndrome induces a long-lasting increase in pancreatic beta-cell mass. Histol. Histopathol., 30(4):479-87, 2015.

Pories, W. J.; Swanson, M. S.; MacDonald, K. G.; Long, S. B.; Morris, P. G.; Brown, B. M.; Barakat, H. A.; de Ramon, R. A.; Israel, G. \& Dolezal, J. M. Who would have thought it? An operation proves to be the most effective therapy for adult-onset diabetes mellitus. Ann. Surg., 222(3):339-50, 1995.

Rubino, F. \& Marescaux, J. Effect of duodenal-jejunal exclusion in a non-obese animal model of type 2 diabetes: a new perspective for an old disease. Ann. Surg., 239(1):1-11, 2004.

Rubino, F.; Forgione, A.; Cummings, D. E.; Vix, M.; Gnuli, D.; Mingrone, G.; Castagneto, M. \& Marescaux, J. The mechanism of diabetes control after gastrointestinal bypass surgery reveals a role of the proximal small intestine in the pathophysiology of type 2 diabetes. Ann. Surg., 244(5):741-9, 2006.

Thaler, J. P. \& Cummings, D. E. Minireview: Hormonal and metabolic mechanisms of diabetes remission after gastrointestinal surgery. Endocrinology, 150(6):2518-25, 2009.

Tian, L. \& Jin, T. The incretin hormone GLP-1 and mechanisms underlying its secretion. J. Diabetes, 8(6):753-65, 2016.

Wilson-Pérez, H. E.; Chambers, A. P.; Ryan, K. K.; Li, B.; Sandoval, D. A.; Stoffers, D.; Drucker, D. J.; Pérez-Tilve, D. \& Seeley, R. J. Vertical sleeve gastrectomy is effective in two genetic mouse models of glucagon-like Peptide 1 receptor deficiency. Diabetes, 62:2380-5, 2013.

Yabe, D. \& Seino, Y. Two incretin hormones GLP-1 and GIP: comparison of their actions in insulin secretion and b cell preservation. Prog. Biophys. Mol. Biol., 107(2):248-56, 2011.

Yousseif, A.; Emmanuel, J.; Karra, E.; Millet, Q.; Elkalaawy, M.; Jenkinson, A. D.; Hashemi, M.; Adamo, M.; Finer, N.; Fiennes, A. G.; Withers, D. J. \& Batterham, R. L. Differential effects of laparoscopic sleeve gastrectomy and laparoscopic gastric bypass on appetite, circulating acyl-ghrelin, peptide YY3-36 and active GLP-1 levels in non-diabetic humans. Obes. Surg., 24(2):241-52, 2014.

\section{Corresponding author: \\ Dr. JA Prada-Oliveira}

Department of Human Anatomy and Embryology

Faculty of Medicine

Plaza Fragela s/n.

University of Cádiz

Cádiz, 11003

ESPAÑA

\section{Email: arturo.prada@uca.es}

Received : 02-05-2018

Accepted : 17-09-2018 